\title{
Loops: Twisting and Scaling
}

\author{
Robert W. Walsh \\ Centre for Astrophysics, University of Central Lancashire, \\ Preston, Lancashire PR1 2HE, UK
}

\begin{abstract}
Loop-like structures are the fundamental magnetic building blocks of the solar atmosphere. Recent space-based EUV and X-ray satellite observations (from Yohkoh, SOHO and TRACE) have challenged the view that these features are simply static, gravitationally stratified plasma pipes. Rather, it is now surmised that each loop may consist of a bundle of fine plasma threads that are twisted around one another and can brighten independently. This invited paper will outline the latest developments in "untangling" the topology of these features through observational analysis and magnetohydrodynamic modelling. In particular, recent interest has centred on how the observed thermal profile along loops can be employed as a tool to diagnose any localised energy input to the structure and hence constrain the presence of a particular coronal heating mechanism. The implications of superior resolution plasma thread observations (whether spatial, temporal or spectral) from future space missions (SolarB, STEREO, Solar Dynamics Observatory and Solar Orbiter) will be discussed.
\end{abstract}

\section{Introduction}

"...the appearance of jets of liquid fire, rising and falling in graceful parabolas", C.A. Young, The Sun, 1895

The discovery that a significant proportion of the radiation emitted from the solar corona is concentrated along well-defined loops represented a major advance in our understanding of the Sun. These loops are the basic structural elements of this environment (Vaiana and Rosner, 1978). The complex and often subtle magnetic field and plasma interactions believed to be occurring in these features have spawned a multitude of theoretical studies as well as being the aim of recent solar observational programmes. The Yohkoh, SOHO and TRACE missions have revealed loops in unprecedented detail; for example, Figure 1a displays a SOHO/MDI magnetogram while Figure $1 \mathrm{~b}$ is a simultaneous SOHO/EIT EUV $171 \AA$ (peak temperature $\approx 1 \mathrm{MK}$ ) image of the corona. It can be seen that the regions of brightest EUV emission (active regions) correspond to the areas of strongest magnetic field. It is now believed universally that EUV loops which constitute this region coincide with magnetic flux tubes and occur as the plasma and thermal energy can flow along but not easily across the magnetic field. 

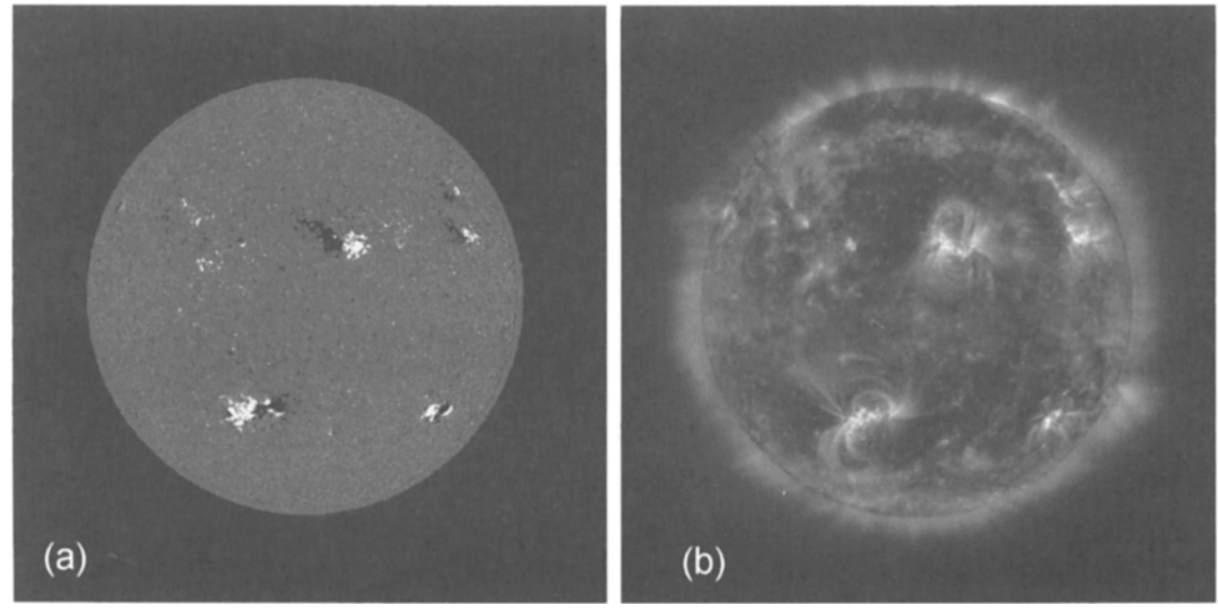

Figure 1. Simultaneous (a) SOHO/MDI magnetogram (white(black) indicates the line-of-sight component of the magnetic field out of (in to) the Sun) and (b) SOHO/EIT $171 \AA$ image.

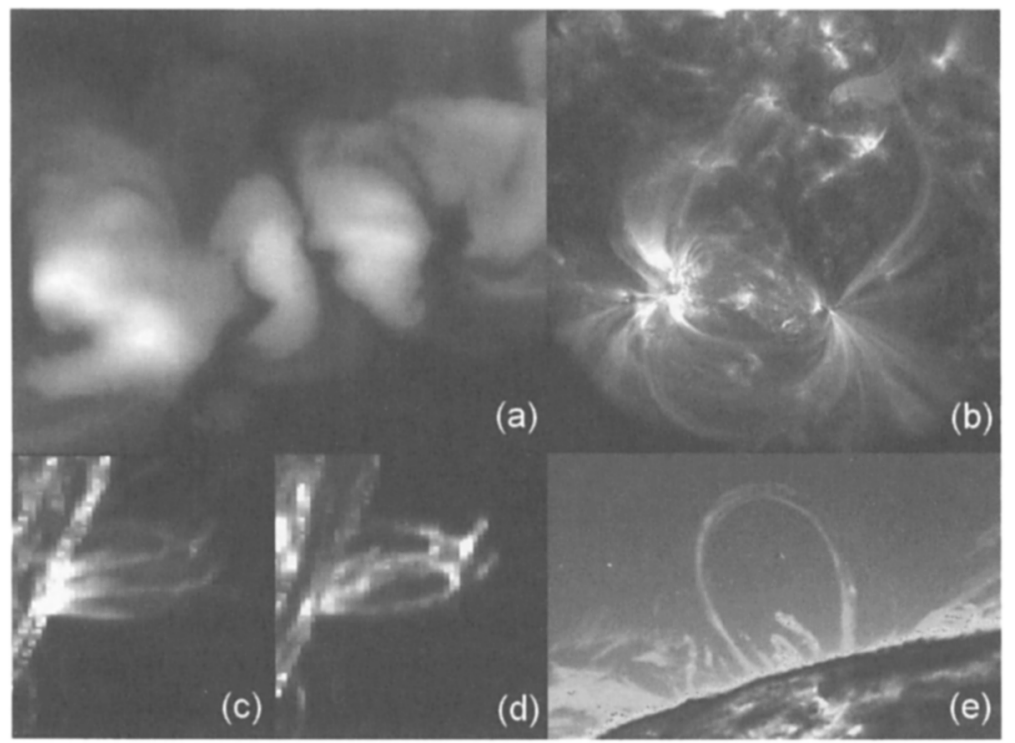

Figure 2. Solar loop-like structures as observed in (a) soft x-rays by Yohkoh/SXT; (b) Fe IX-X 171 by TRACE; (b) Ov by SOHO/CDS; (d) HeI by SOHO/CDS and (e) Lyman alpha by TRACE. 
With this is mind, it must be noted that the phrase "loop" is an inclusive, general term. Firstly, one must consider the fact that "loops" are observed over an extensive temperature range. Figure 2 displays loops in soft x-rays from Yohkoh (a; > $2 \mathrm{MK}$ ), in EUV from SOHO and TRACE (b, c, d in Fe IX-X $(\approx 1 \mathrm{MK}), \mathrm{Ov}(250,000 \mathrm{~K})$ and $\mathrm{HeI}(20,000 \mathrm{~K})$ respectively) and in Lyman $\alpha$ (TRACE $1216 \AA, 10,000 \mathrm{~K}$ ). Are all these loops the same phenomena, with the same physical processes operating in each of them (such as the elusive heating mechanism)?

Secondly, loops appear over wide spatial scales. Transequatorial loops span a substantial portion of the solar disc (e.g., Glover et al. 2003), while some EUV bright points have been observed to show small, arch-like flows (Ireland et al., 1999). Similarly, there is the discussion as to whether an individual loop has yet to be resolved. Is there a difference in the dominant physics operating in "fatter" Yohkoh/SXT loops (Figure 1a) than in the thread-like plasma strands observed in EUV by TRACE (Figure 1b)? Or is it simply a matter of instrumental resolution?

Thirdly, we must not ignore the time-dependent nature of the loops we observe, whether these are flare loops cooling down (Warren et al. 2002) or the numerous small-scale loop brightenings and flows observed during normal active region evolution (Nightingale et al., 1999). Can all of the above be described by a single coronal loop theory?

In light of the above, this review will concentrate only upon hot loops (> $1 \mathrm{MK}$ ). Recently, there has been a substantial amount of interest in these structures and this is discussed below by considering their magnetic topology and then investigating the properties of individual plasma strands.

\section{Magnetic Topology}

In endeavouring to untangle the topology of loops, there exists currently an observational trade-off. Firstly, if we observe an active region on the limb then we can, for example, measure the height of the loops above the solar surface. However, this is at the expense of having a "side-on" image and no directly observable magnetic field information. On the other hand, observations at disk centre provide the opportunity of obtaining excellent line-of-sight photospheric magnetograms but in EUV say, you are now looking down directly on the region and thus have little information about loop height. This situation is further complicated by the optically thin nature of the corona; that is, as you peer through the solar atmosphere, the emission you receive is the summed effect of the entire plasma volume which is radiating along your line of sight.

In the magnetically dominant environment of the corona, magnetic pressure dominates plasma pressure and thus, an equilibrium structure with no flow obeys a magnetohydrostatic force balance equation;

$$
0=-\nabla p+\mathbf{j} \times \mathbf{B}+\rho \mathbf{g}
$$

where $\mathbf{B}$ is the magnetic field strength with $\nabla \cdot \mathbf{B}=0 ; p$ is the plasma pressure; $\rho$ is the plasma density; $g$ is the gravitational acceleration at the Sun's surface and $\mathbf{j}=\nabla \times \mathbf{B} / \mu$ is the electric current (with $\mu$ the magnetic permeability). If 
the pressure gradients and gravity are negligible, we have

$$
0=\mathbf{j} \times \mathbf{B}
$$

so that the $\mathbf{j}$ must be parallel to the $\mathbf{B}$. Consequently,

$$
\nabla \times \mathbf{B}=\alpha \mathbf{B}
$$

where $\alpha$ is a function of position within a force-free magnetic field. Taking the divergence of (3) we find B. $\nabla \alpha=0$. This means that the rate of change of $\alpha$ in the direction of the magnetic field is zero or rather $\alpha$ is constant along a given field line (but can change from one field line to the next). If we assume that $\alpha$ is uniform everywhere then the curl of (3) gives

$$
\left(\nabla^{2}+\alpha^{2}\right) \mathbf{B}=0
$$

These are called constant $\alpha$ or linear force-free fields. Numerous authors use this approach to not only calculate the three-dimensional nature of magnetic loops in active regions (e.g., Falconer et al. 2000) but also to examine the toplogy of filaments and flares (Aulanier et al. 2000) and to estimate plasma scaling laws (Mandrini et al. 2000). Also, non-constant $\alpha$ models are emerging (Régnier et al. 2002).

The three-dimensional magnetic topology model displayed in Figure 3 was constructed as follows. Coincident MDI magnetogram and CDS EUV Mg IX images of a bright active region close to disk centre are taken. The magnetogram is used as the boundary condition for the normal component of the magnetic field through the bottom boundary of your numerical box. The value of $\alpha$ is chosen by estimating the best fit between the extrapolated field lines and the bright loops in the EUV image. Hence, the three-dimensional nature of the field is calculated. However, it must be remembered that the entire region is filled with magnetic field. The field lines drawn in Figure 3 are only the ones that correspond to the flux tubes that are filled with plasma radiating at the observed temperature. Different loops at different temperatures in the active region may require different $\alpha$ values to produce a good match. This is the problem of using a force-free model. Also, this model does not reveal why these specific loops should radiate in this way.

It must be remembered that these loop features are rooted into the so-called "magnetic carpet" of the photosphere with individual flux elements appearing to have a lifetime of about 14 hours (Hagenaar 2001). The fact that an individual flux fragment can, on average, only be tracked for such a short time period of time, has significant implications for the overlying magnetic topology. With this in mind, Priest et al. (2002) have examined a "coronal tectonics" model where each coronal loop we can observe is actually connected to many intense, smaller scale flux sources. They argue that even TRACE EUV loops are highly fragmented, consisting of at least ten finer threads. These numerous thread-like connections occur at the boundaries of supergranule cells with some stretching over many cells. Thus, as sketched in Figure 4, the coronal environment is filled with small, intermediate and large magnetic loops, that are continuously jostled around and pressed up against each other in the coronal volume. This leads to 

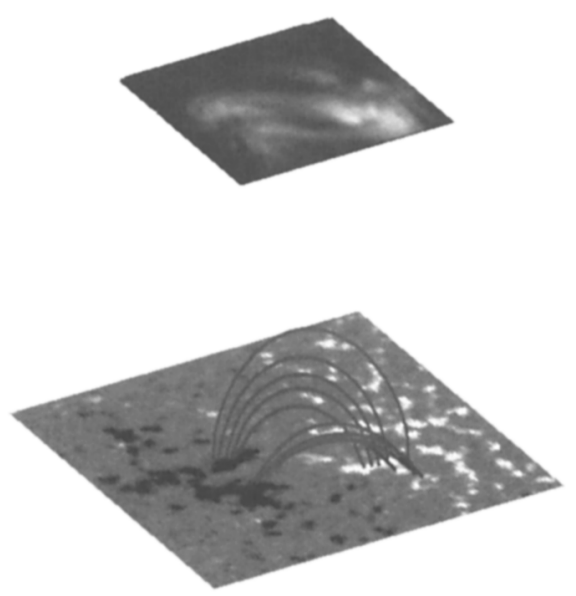

Figure 3. An example of the three-dimensional reconstruction of possible magnetic loop topology using an MDI magnetogram and an EUV $\mathrm{Mg}$ IX image from CDS.

a very complicated, three dimensional, dynamic picture of the magnetic linkage between individual magnetic strands (also see Close et al., 2003, for statistical analysis of the possible flux tube lengths and number of connections from a single flux element predicted by a potential model of the above.).

With this in mind, one way to unwrap such a complex scenerio is to concentrate upon the physics of an individual strand or plasma element. This is outlined in the next Section.

\section{Plasma Dynamics}

One-dimensional hydrodynamic modelling of coronal loops along individual fieldlines has been popular since the late 1970's (Peres 2000). Essentially, there are two approaches. Firstly, a ground-breaking advance in the analysis of active region loops by Rosner et al. (1978) employed Skylab X-ray data and hydrostatic loop modelling to derive two scaling laws. These state that,

$$
T_{\max }=1.4 \times 10^{3}(p L)^{1 / 3} \mathrm{~K},
$$

(where the maximum loop-top temperature $T_{\max }$ is a function of the loop length $L$ (centimetres) and pressure $p\left(\right.$ dyn $\left.\left.\mathrm{cm}^{-2}\right)\right)$ and

$$
E_{H}=9.8 \times 10^{4} p^{7 / 6} L^{-5 / 6} \mathrm{ergs}^{-1} \mathrm{~cm}^{-2},
$$

which comments on the nature of the coronal heating mechanism by calculating the heat deposition rate $E_{H}$. These relationships built an important bridge between observations and theory. Serio et al. (1981) modified the above to take into account pressure and heating deposition scale heights in the corona; more recent updates can be found in Aschwanden and Schrijver (2002). 


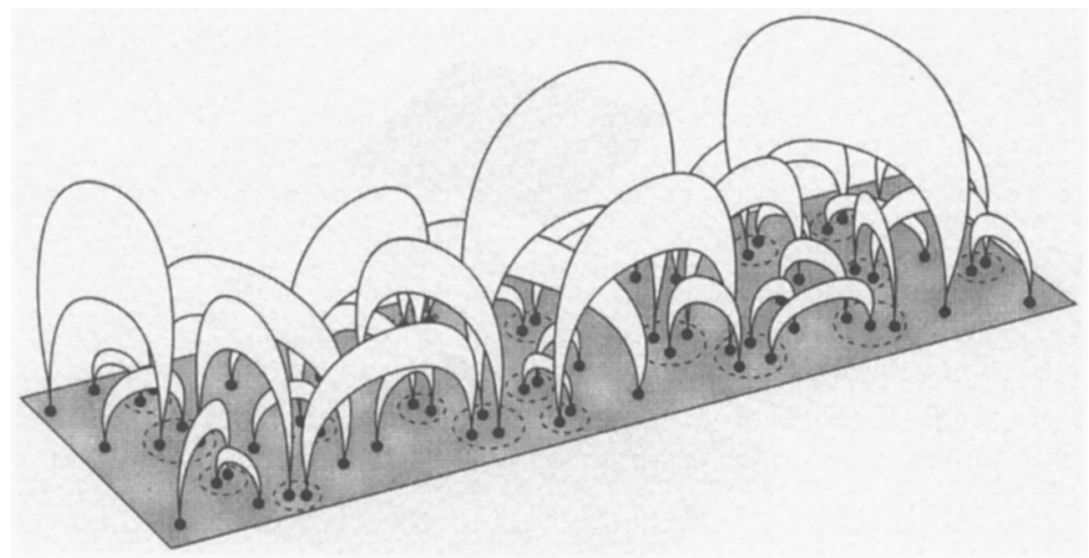

Figure 4. A three-dimensional view of magnetic topology arising from the network indicates that the photospheric flux elements (dashed ovals) are in general connected to a range of neighbours creating a wide range of loop structures (from Priest et al. 2002).

When examining the hydrostatic thermal profile along a coronal magnetic loop, there must be a balance between the energy sinks (thermal conduction and optically thin radiative loss) and sources (heat input by some mechanism) at each point. It should be noted that differing heating mechanisms can produce heating with spatially different forms. Alfvén waves dissipated by phase-mixing or resonant absorption would tend to heat preferentially close to the loop summit where the amplitude of the fundamental mode would be largest. Also, if a loop broadened substantially at its apex, flux braiding would tend to accumulate there and so give enhanced heating at that location. Loops with uniform crosssections (Klimchuk 2000) could give a more uniform braiding (and therefore heating) profile.

Consider a thin plasma strand observed in the corona as being modelled along a single magnetic field line; neglecting gravity yields the thermal equilibrium equation,

$$
\frac{\partial}{\partial s}\left(\kappa_{\|} \frac{\partial T}{\partial s}\right)=\frac{\rho^{2}}{\tilde{\mu} R} \chi T^{\alpha}-H(s),
$$

where $T$ is the temperature; $s$ is the distance along the loop; $\rho$ is the density; $\kappa_{\|}=\kappa_{0} T^{5 / 2} \mathrm{~W} \mathrm{~m}^{-1} \mathrm{~K}^{-1}$ is the coefficient of thermal conductivity parallel to the field with $\kappa_{0}=10^{-11}$ for the corona; $R$ is the molar gas constant $\left(8.3 \times 10^{3}\right.$ $\mathrm{m}^{2} \mathrm{~s}^{-2} \mathrm{~K}^{-1}$ ) and $\tilde{\mu}$ is the mean molecular weight with $\tilde{\mu}=0.6$ in the ionized corona. The first term on the right-hand side of equation (5) is the effect of the radiative loss (e.g., Cook et al. 1989). The function $H$ is the spatially dependent energy input to the system.

For the hot loop solutions we are investigating (Walsh, 1999), we require temperature profiles with a hot summit temperature $\left(>10^{6} \mathrm{~K}\right)$ and cool footpoints embedded in the chromosphere $\left(\approx 10^{4} \mathrm{~K}\right)$. Assuming that the loop is symmetrical about the loop apex $(d T / d s=0)$, Figure 5 displays the dependence of the thermal structure on how this energy is distributed for a $60 \mathrm{Mm}$ coronal 

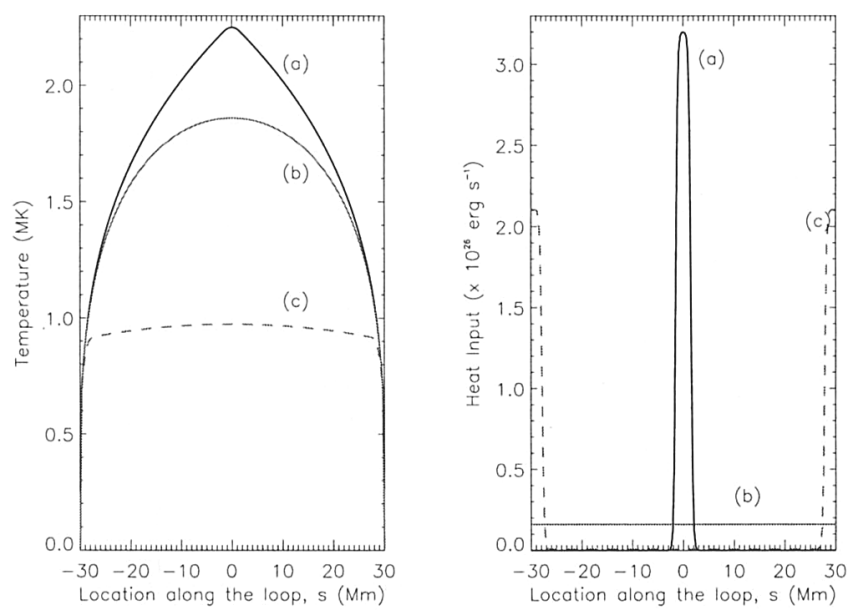

Figure 5. Comparison of $T(s)$ with $H(s)$ where the total energy input to the loop structure remains constant.

loop (-30 Mm $\leq s \leq 30 \mathrm{Mm})$ with a total fixed amount of energy being deposited $\left(1.6 \times 10^{25} \mathrm{erg} \mathrm{s}^{-1}\right)$; i.e., the total amount of energy being deposited in the loop is exactly the same irrespective of how the energy is distributed spatially.

If heating is applied uniformly along the loop (b), an apex temperature of about $1.85 \times 10^{6} \mathrm{~K}$ results. However, if the heat is deposited predominantly at the apex in the rarer coronal part of the loop (a), the temperature at this point is higher $\left(2.25 \times 10^{6} \mathrm{~K}\right)$ than in the uniform case. The temperature gradient along the loop has increased and therefore conduction plays a vital role in redistributing the heat. If the same total amount of energy is released preferentially at the base of the loop (c), the thermal profile becomes very flat with an apex temperature dropping in value to about $10^{6} \mathrm{~K}$. In this case, conduction is greatly reduced in the coronal part of the loop while most of the deposited energy is radiated away. Thus, it is evident that the location of the dominant heat input to the plasma thread has an important and possibly observable effect.

Thus, there has been a recent concentration of effort in determining the temperature structure along magnetic loops. The two avenues of investigation have been to (i) calculate loop thermal profiles from observations and then match this with a $T(s)$ from a 1D hydrostatic model that will yield a unique $H(s)$, or (ii) fold your model calculations through some instrument response function (such as the TRACE EUV filters) and compare the results with observations. However, these approaches have proved out to not be as straightforward as it would first appear.

Priest et al. (2000) introduces a hydrostatic loop model that balances only thermal conduction and $H(s)$. Comparing the model with coronal temperature measurements along a large, diffuse, soft x-ray loop, they find that a spatially uniform heat input provides the best fit to the data. However, Mackay et al. (2000) have challenged this analysis, stating that the conclusion of uniform heating lacks statistical significance; their results point toward the energy being deposited close to the loop foot-points. Also Reale (2002) reanalyses the same 
loop and after background subtraction is undertaken, deduces that the loop is heated at the apex. The fact that three different data interpretation techniques can come to different conclusions about the same dataset is alarming.

The controversy continues over into analysing EUV loops. Aschwanden et al. $(2000 \mathrm{~b}, 2001)$ use filter ratios of SOHO/EIT and TRACE EUV bands (171 $\AA$ to $195 \AA$ say) to argue that the near isothermal loops observed indicate an $H(s)$ weighted towards heating at the loop base. However, it should be noted that the filter-ratio technique has been criticized as being too simplistic and that the concept of the differential emission measure (DEM) must be employed as a means of calculating the amount of emitting material present in an optically thin environment. Thus, Schmelz et al. (2001) derive a DEM distribution for several pixels along an isolated loop using spectral-line data from SOHO/CDS and broadband data from Yohkoh/SXT; they find that the calculated $T(s)$ is clearly inconsistent with an isothermal (and hence footpoint heated) loop. Schmelz (2002) takes the analysis further by reproducing what SOHO/EIT would observe of the aforementioned SOHO/CDS loop and obtains an isothermal structure. Martens et al. (2002) explains that the TRACE isothermal results are due to the existence of a broad, flat plateau in the DEM from $\approx 0.7 \mathrm{MK}$ to $2.8 \mathrm{MK}$; since SOHO/EIT and TRACE narrowband filters fall within this range, this leads to an isothermal ratio. Testa et al. (2002) argue as well that diagnostics by use of filter ratios are ambiguous as their TRACE temperature calculations yield both very hot $(>5 \mathrm{MK})$ and cool $\left(\approx 10^{5} \mathrm{~K}\right)$ loops. Recently, Chae et al. (2002) argue that by comparing two filter ratios simultaneously (171 $\AA$ to 195 $\AA$ and $195 \AA$ to $284 \AA$ ), an unambiguous temperature can be found.

It should also be noted that the problem of determining $T(s)$ is compounded when using a rastering spectrometer like the Normal Incidence Spectrometer (NIS) on SOHO/CDS. The NIS slit is exposed for a time over the target while it covers the field from right to left in discrete steps to build up an image. However, if plasma is evolving in some way while CDS is rastering, a spatiotemporal smearing of the diagnosed plasma parameters occurs (Walsh 2002). The extent of this smearing depends upon the timescale of the plasma changes themselves relative to the exposure time employed and the orientation of the loop on the Sun relative to the position of the moving vertical slit. One way this could be overcome is to set the slit at one location in the loop (apex or footpoint say) and track the temperature/density changes at that point. This could indicate the possible heating timescale operating in a specific loop.

\section{Conclusions and Questions}

“...the corona has a very complex structure, it appears to be formed of entangled arches...", B. Lyot, 1944

An incredible amount of new information about solar loop-like structures has been obtained over the last decade. Observational analysis and theoretical modelling of these magnetic building blocks of the solar atmosphere is advancing but there are still several basic questions that need to be answered.

Firstly, although the corona is filled with magnetic field, why do certain magnetic strands appear at certain temperatures? Possibly this is due to varia- 
tions (in space, time and quantity) of the energy deposition - since the ultimate source of the coronal heating is the magnetic field, a link must be made between the observed emission in loops and the magnetic field. Several authors have attempted to achieve this; Golub et al. (1980) uses Skylab soft X-ray data and ground-based magnetograms while Fisher et al. (1998) employs Yohkoh/SXT and ground-based vector magnetograms to demonstrate that the X-ray flux varies as $\phi^{1.19}$, where $\phi$ is the total unsigned magnetic flux of the active region. Fludra and Ireland (2003) assume that the radiance of EUV line Fe XVI $360.76 \AA(\approx 2 \mathrm{MK})$ in an individual coronal loop is related by a power law to the footpoint magnetic field. These authors use a Laplace-transform approach to sum the distribution of field elements and derive the exponent of the power-law relating to the EUV flux magnetic field. However, we are someway off identifying why a specific plasma thread "lights up" in OV say, rather than in Mg IX within an active region. Thus, it is vital that the physical plasma parameters along a wide range of loop-like structures are obtained.The EIS instrument on Solar-B will make a major contribution to this area.

Secondly, what is the three dimensional topology of these loops? From a planar image, it is difficult to determine the basic three-dimensional geometry of the loop. For example; what is the length, inclination to the solar surface or aspect ratio? These are all important quantities required for modelling. Attempts have been made to deal with this problem (e.g., Aschwanden et al. 1999, 2000a) but eventually true three-dimensional imaging is required. This will be addressed by the STEREO mission.

Thirdly, have we resolved spatially the basic structural element of a loop? For example, Lenz et al. (1999) deduced from long-lived TRACE loops that the structures may consist of a bundle of filamentary loop threads at a range of temperatures which, when averaged over, give the appearance of isothermal loops. In that regard, Figure 6 displays a simultaneous images of the same portion of the solar limb taken by CDS in the Mg IX line (1 MK) and by TRACE $171 \AA$. The close-up of a small area on the right-hand side demonstrates clearly that the filamentary nature of the corona observed by TRACE is averaged out by the CDS pixel resolution. Within every CDS pixel, there may be multiple, multi-thermal plasma strands which could be interweaved with one another. Thus even the DEM observations from CDS will be the spatial average across a bundle of plasma threads. With the current instrument specifications for future solar missions, it appears that the solar community will have to wait for the proposed instrumental resolution onboard Solar Orbiter $(75$ (35) km on the Sun for the spectrometer (partial field imager)) to have the possibility of imaging finer plasma strands.

Fourthly, how dynamic are these plasma strands? Even at current temporal resolution limits (tens of seconds), we see small scale brightenings and evolving plasma flows in loops (Winebarger et al. 2002). Thus, taking a thermal snapshot of a plasma strand and fitting an hydrostatic model through the data-points is but a first step. A time series of thermal profiles would be more appropriate. This important aspect has been recognized by several authors. For example, Reale et al. (2000b) use a hydrodynamic model to simulate the temporal evolution of coronal plasma along a particular loop observed by TRACE (Reale et al. 2000a). Warren et al. (2002) investigate bright EUV loops that persist longer 

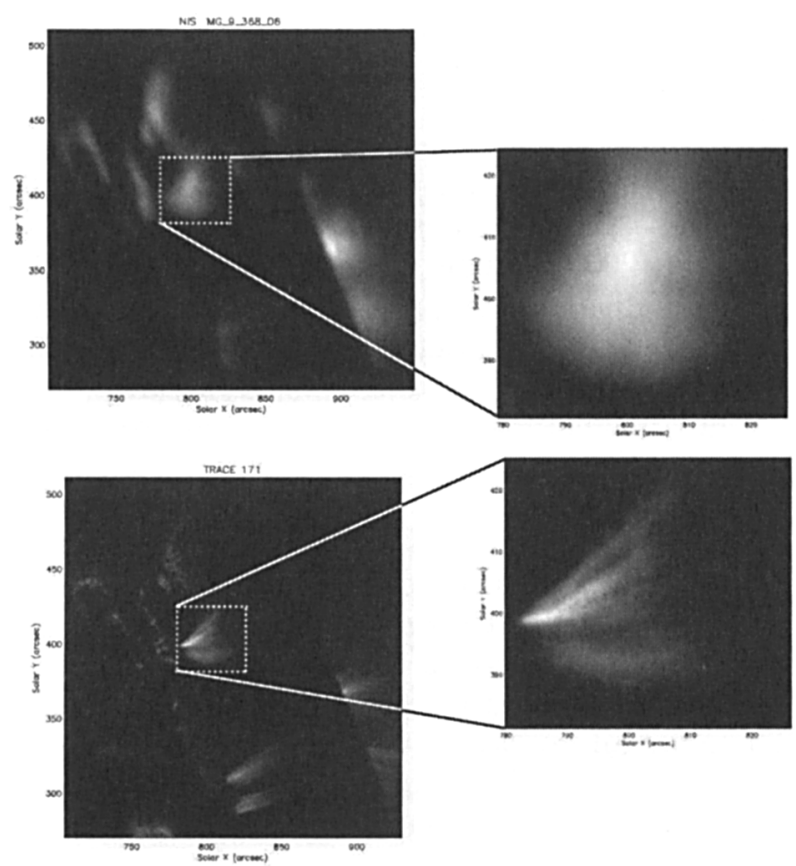

Figure 6. Simultaneous images taken by SOHO/CDS (in the Mg-IX line at a temperature of approximately 1.0 MK) and TRACE 171$\AA$ bandpass (also about $1 \mathrm{MK}$ ). The close-up of the same region on the right demonstrates that the filamentary nature of the corona observed by TRACE is averaged out by the CDS pixel resolution.

than the characteristic radiative cooling time for a $1 \mathrm{MK}$ corona, arguing that their behaviour can be reproduced by a collection of smaller plasma threads that are repeatedly heating and cooling down. Spadaro et al. (2003) show that the problem of overdense loops (Aschwanden et al. 2001), where the observed density is in excess of that predicted by scaling laws, can be explained by having a transient heating burst close to one loop footpoint. Walsh et al. (1997) consider discrete energy bursts over a wide energy $\left(10^{24} \mathrm{erg}\right.$ to $\left.10^{28} \mathrm{erg}\right)$ and temporal ranges and find that the larger events do not occur frequently enough to maintain typical coronal temperatures in a hot loop. The rapid imaging capabilities of the Solar Dynamics Observatory will make significant progress in determining the extent of this time-dependent behaviour.

\section{Acknowledgements}

RWW was supported in this work by a Leverhulme Trust Research Fellowship. SOHO is a project of international cooperation between ESA and NASA. SOHO images are courtesy of SOHO CDS/EIT/MDI consortia. TRACE images are courtesy of the Stanford-Lockheed Institute for Space Research. 


\section{References}

Aschwanden, M.J., Newmark, J. S., \& Delaboudinière, J.-P., Neupert, W.M.; Klimchuk, J. A., Gary, G. A., Portier-Fozzani, F., \& Zucker, A. 1999, ApJ, 515, 842

Aschwanden, M.J., Alexander, D., Hurlburt, N., Newmark, J.S., Neupert, W.M., Klimchuck, J.A., \& Gary, G.A. 2000a, ApJ, 531, 1129

Aschwanden, M.J., Nightingale, R.W., \& Alexander D. 2000b, ApJ, 541, 1059

Aschwanden, M., \& Schrijver, C. J. 2002, ApJS, 142, 269

Aschwanden, M., Schrijver, C. J., \& Alexander, D. 2001, ApJ, 550, 1036

Aulanier, G., \& 11 co-authors 2000, Adv in Space Res., 26, 485

Chae, J., Park, Y.-D., Moon, Y.-J, Wang, H., \& Yun, H.S. 2002 ApJ, 567, L159

Close, R.M., Parnell, C.E., Mackay, D.H., \& Priest, E.R. 2003, Solar Phys., 212, 251

Cook, J.W., Cheng, C.-C., Jacobs, V.L., \& Antiochos, S.K. 1989, ApJ, 338, 1176

Falconer, D.A., Gary, G.A., Moorw, R.L., \& Porter, J.G. 2000, ApJ, 528, 1004

Fisher, G. H., Longcope, D. W., Metcalf, T. R., \& Pevtsov, A. 1998 ApJ, 508, 885

Fludra, A., \& Ireland, J. 2003, A\&A, 398, 297

Glover, A., Harra, L.K., Matthews, S.A., \& Foley, C.A. 2003, A\&A, 400, 759

Golub, L., Maxson, C., Rosner, R., Serio, S., \& Vaiana, G.S. 1980, ApJ, 238, 343

Hagenaar, H.I. 2001, ApJ, 555, 448

Ireland, J., Wills-Davey, M., \& Walsh, R. W. 1999, Solar Phys., 190, 207

Klimchuk, J.A. 2000, Solar Phys., 193, 53

Lenz, D.D., DeLuca, E.E., Golub, L., Rosner, R., Bookbinder, J. A., Litwin, C., Reale, F., \& Peres, G. 1999, Solar Phys., 190, 131

Mackay, D.M., Galsgaard, K., Priest, E. R., \& Foley, C. R. 2000, Solar Phys. 193,93

Mandrini, C. H., Démoulin, P., \& Klimchuk, J. A. 2000, ApJ, 530, 999

Martens, P.C.H., Cirtain, J.W., \& Schmelz, J.T. 2002, ApJ, 577, L115

Nightingale, R.W., Aschwanden, M.J., \& Hurlburt, N.E. 1999, Solar Phys., 190, 249

Peres, G. 2000, Solar Phys., 193, 33

Priest, E.R., Foley, C. R., Heyvaerts, J., Arber, T. D., Mackay, D., Culhane, J. L., \& Acton, L. W. 2000, ApJ, 539, 1002

Priest, E.R., Heyvaerts, J., \& Title, A. 2002, ApJ, 576, 533

Reale, F. 2002, ApJ, 580, 566

Reale, F., Peres, G., Serio, S., Betta, R.M., DeLuca, E.E., \& Golub, L. 2000a, ApJ, 535, 412

Reale, F., Peres, G., Serio, S., Betta, R.M., DeLuca, E.E., \& Golub, L. 2000b, ApJ, 535, 423

Régnier, S., Amari, T., \& Kersalé, E. 2002, A\&A, 392, 1119 
Rosner, R., Tucker, W. H., \& Vaiana, G. S. 1978, ApJ, 220, 643

Schmelz, J.T., Scopes, R.T., Cirtain, J.W., Winter, H.D., \& Allen, J.D. 2001, ApJ, 556, 896

Schmelz, J.T. 2002, ApJ, 578, L161

Serio, S., Peres, G., Vaiana, G. S., Golub, L., \& Rosner, R. 1981, ApJ, 243, 288

Spadaro, D., Lanza, A.F., Lanzafame, A.C., Karpen, J.T., Antiochos, S.K., Klimchuk, J.A., \& MacNeice, P.J. 2003, ApJ, 582, 486

Testa, P., Peres, G., Reale, F., \& Orlando, S. 2002, ApJ, 580, 1159

Vaiana, G.S., \& Rosner, R. 1978, ARA\&A, 16, 393

Walsh, R. W. 1999, ESA SP-446, 687

Walsh, R. W. 2002, ESA SP-508, 335

Walsh, R. W., Bell, G.E., \& Hood, A.W. 1997, Solar Phys., 171, 81

Warren, H.P., Winebarger, A.R., \& Hamilton, P.S. 2002, ApJ, 579, L41

Winebarger, A.R., Warren, H.P., Van Ballegooijen, A., DeLuca, E.E., \& Golub, L. 2002, ApJ, 567, L89 